\title{
Melanoma-Associated Spongiform Scleropathy in Oculodermal Melanocytosis with Primary Orbital Melanoma
}

\author{
Roshni U. Ranjit ${ }^{a} \quad$ llya M. Leyngold ${ }^{a}$ Curtis E. Margo ${ }^{a, b}$ \\ Departments of a Ophthalmology and ${ }^{b}$ Pathology and Cell Biology, Morsani College of Medicine, University of \\ South Florida, Tampa, Fla., USA
}

\section{Key Words}

Melanoma-associated spongiform scleropathy · Melanosis oculi · Nevus of Ota $\cdot$ Oculodermal melanosis $\cdot$ Scleral degeneration

\begin{abstract}
Purpose: To describe spongiform scleropathy in a patient with oculodermal melanosis and without evidence of uveal melanoma. Methods: Clinical-pathological correlation conducted in compliance with HIPPA (Health Insurance Privacy and Portability Act) regulations. Results: Melanoma-associated spongiform scleropathy was an incidental finding in an 87-year-old woman with oculodermal melanocytosis treated for primary orbital melanoma. All previously reported cases of this scleropathy have been associated with uveal melanoma. Conclusions: The mechanism of scleral degeneration in melanoma-associated spongiform scleropathy is unknown, and its clinical and prognostic significance is speculative. This is the first case of a so-called melanoma-associated spongiform scleropathy reported in an eye without uveal melanoma.

(c) 2016 S. Karger AG, Basel
\end{abstract}

\section{Introduction}

Melanoma-associated spongiform scleropathy is a noninflammatory degenerative change reported exclusively with choroidal or ciliary body melanoma [1]. The first description of this condition is attributed to Stefani at the European Ophthalmic Society meeting in 1989 [2]. Subsequent studies have shown that melanoma-associated spongiform scleropathy occurs in areas of direct sclera contact with uveal melanoma. This abnormality has been reported in $11 \%$ to one third of eyes with posterior uveal melanoma [1-3]. Scleral aberration may have been overlooked by pathologists in the past, because the collagen alterations were attributed to fixation or processing artifact. Although its pathogenesis remains obscure, preliminary evidence suggests that the degenerative change may enhance the risk of scleral invasion and extrascleral spread [1-4]. We report a patient with oculodermal melanocytosis (or nevus of Ota) who developed an ipsilateral primary orbital melanoma. An incidental histologic finding (and subject of the paper) was melanoma-associated spongiform scleropathy contiguous to the diffuse choroidal nevus. We believe this is the first report of melanoma-associated spongiform scleropathy found in an eye without uveal melanoma. This study was conducted in compliance with HIPPA (Health Insurance Privacy and Portability Act) regulations.

\section{KARGER}

E-Mail karger@karger.com www.karger.com/oop
(C) 2016 S. Karger AG, Basel

2296-4681/16/0024-0276\$39.50/0
Curtis E. Margo, MD, MPH

Department of Ophthalmology, Morsani College of Medicine 12901 Bruce B. Downs Blvd.

Tampa, FL 33612 (USA)

E-Mail cmargo@ health.usf.edu 


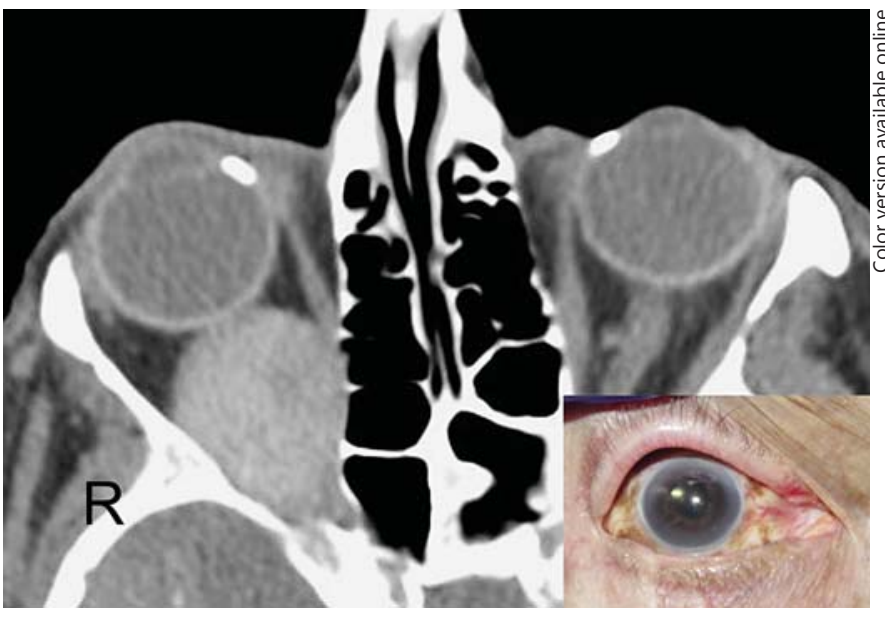

Fig. 1. Axial computed tomography showing a large intraconal tumor extending to the apex of the right orbit. Inset With the lid elevated, brown episcleral pigmentation is seen.

\section{Case Report}

An 87-year-old white woman was referred to an ophthalmologist with a 3-week history of right eye pain. An accompanying computed tomography scan revealed a $3.1 \times 2.2 \times 2.3 \mathrm{~cm}$ right, intraconal orbital mass. The tumor extended to the apex of the orbit; it was hyperdense with minimal enhancement (fig. 1).

Relevant medical history included tan birthmarks of her right eyelids and eye (fig. 1, inset). She had undergone cataract surgery in both eyes years ago. Initial examination showed complete right upper eyelid ptosis, reduced ductions in all directions of gaze, no light perception vision OD with an afferent pupillary defect, $4 \mathrm{~mm}$ proptosis, and elevated intraocular pressure of $39 \mathrm{~mm} \mathrm{Hg}$. Visual acuity was 20/40 in the left eye, with an intraocular pressure of 14 $\mathrm{mm} \mathrm{Hg}$. Slit lamp examination was significant for patches of brown episcleral pigment and a dark brown iris OD.

A biopsy of the anterior edge of the tumor via anterior orbitotomy revealed a heavily pigmented melanoma. An eyelid biopsy showed benign spindle dermal melanocytes (blue nevus). Systemic evaluation revealed no evidence of melanoma (or other malignancy) elsewhere. The patient opted for orbital exenteration.

\section{Pathology}

The posterior orbital tumor was grossly black. The entire optic nerve was easily separated from the tumor and submitted separately for microscopic examination. Histologically, the melanoma consisted of large atypical melanocytes. The tumor invaded surrounding connective tissues and came within several millimeters of the sclera but was never in direct contact with it (fig. 2). Cross-section of the optic nerve was examined at multiple levels (fig. 2, inset). The optic nerve sheath did not contain melanocytes, although several microscopic foci of metastatic melanoma were found in the nerve itself. Collections of bland spindle melanocytes were found in orbital soft tissue near the melanoma. The tumor was present at the posterior surgical margin. Relevant findings in the eye included a diffuse uveal nevus, spindle cell type, involving the iris, ciliary body

Melanoma-Associated Spongiform

Scleropathy in Oculodermal

Melanocytosis

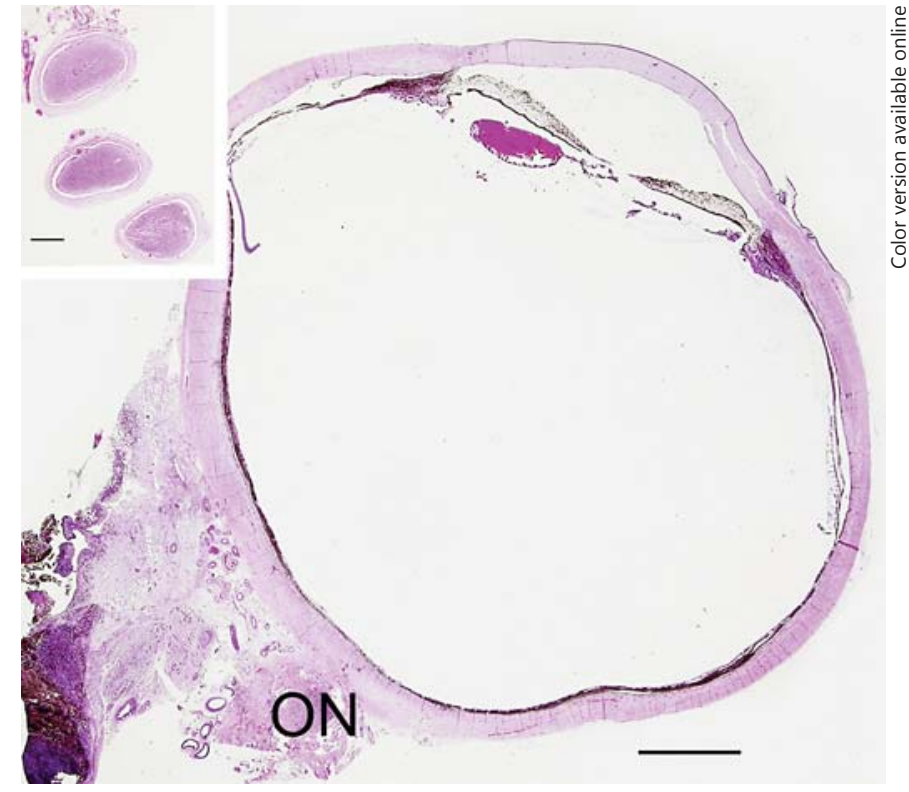

Fig. 2. Low magnified view of the eye and retrobulbar connective tissue showing melanoma in the lower left corner. This is as close as the orbital melanoma approached the globe. The retina was detached during processing. The choroid is modestly thick and heavily pigmented. Scleral abnormalities cannot be appreciated at this magnification (hematoxylin-eosin; bar $=1,600 \mu \mathrm{m}$ ). Inset Crosssections of the optic nerve reveal no direct tumor involvement or heterotopic melanocytes (hematoxylin-eosin; bar $=900 \mu \mathrm{m}$ ).

and choroid (fig. 3). Melanin-bleached sections revealed thin, bland, spindle-shaped nuclei of benign melanocytes (fig. 4). No mitotic figures were observed. A substantial portion of the inner sclera in contact with the uveal tract (roughly $80 \%$ ) revealed spongiform degeneration of collagen characterized by feathery or shredded wheat appearance of longitudinally cut fibers (fig. 3). The areas of spongiform degeneration contiguous with the uveal tract tended to involve less than a third of scleral thickness (i.e. inner third), and stained subtlety less intensely with alcian blue and colloidal iron than normal surrounding sclera. Benign spindle melanocytes were widely and haphazardly scattered throughout the sclera. The regional density of scleral melanocytes did not correlate with the presence of melanoma-associated spongiform scleropathy.

\section{Discussion}

Oculodermal melanocytosis is a hamartomatous malformation of melanocytes involving soft tissues of first and second divisions of the trigeminal nerve [5]. Benign spindle and dendritic melanocytes are found in varying concentrations within the dermis, substantial propria of conjunctiva, episclera, sclera, and uveal tract. Although 

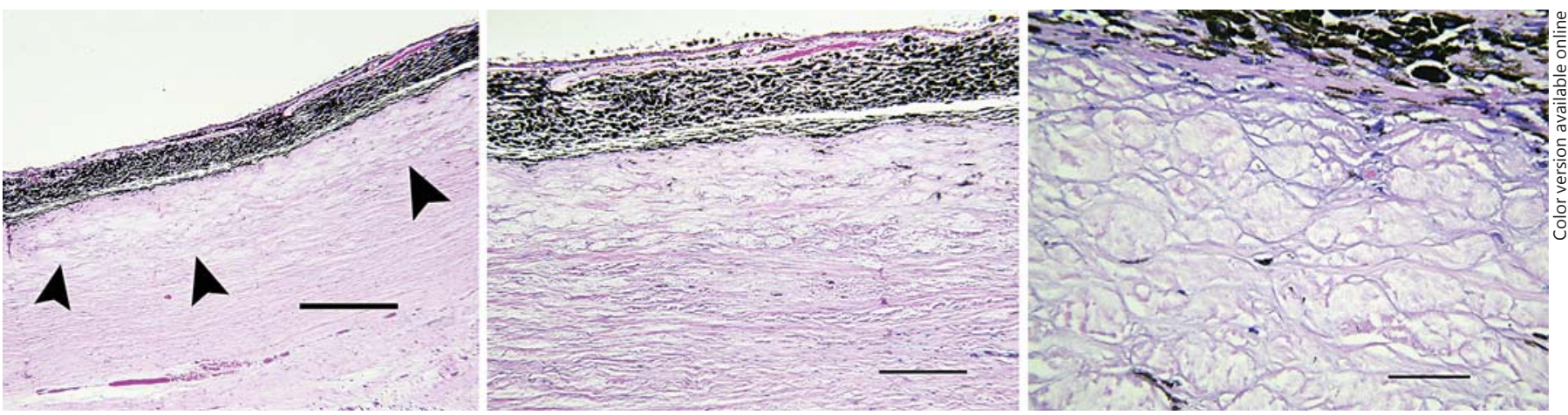

Fig. 3. Three hematoxylin-eosin-stained sections showing melanoma-associated spongiform scleropathy involving the inner third of the sclera. The left panel shows a heavily pigmented, uniformly thick nevus of the choroid and abnormal inner scleral collagen (arrowheads; bar $=300 \mu \mathrm{m})$. The middle panel at higher magnifica-

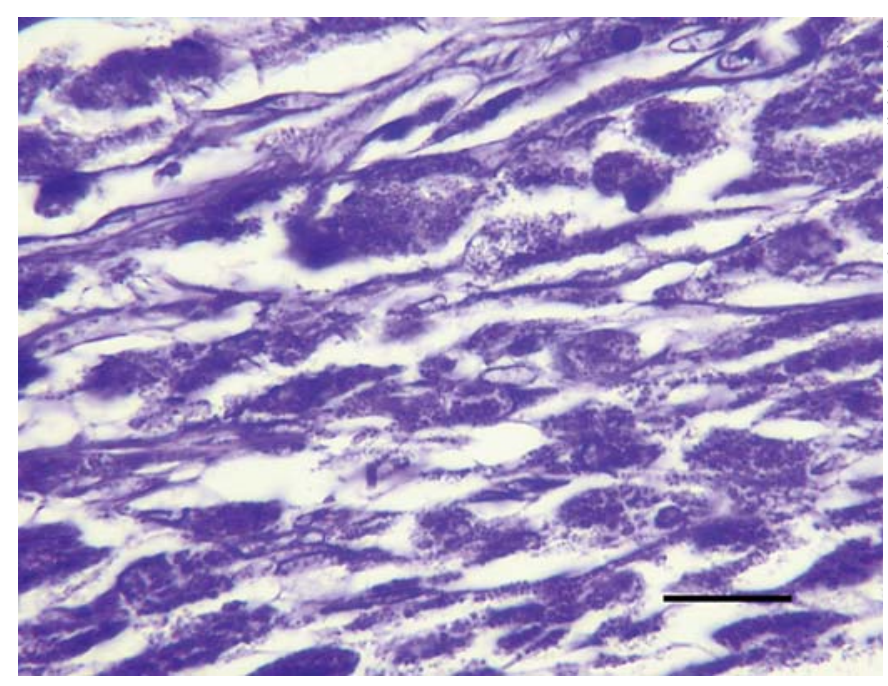

Fig. 4. The melanin-bleached section from the choroid showing faint nuclei of thin, spindle-shaped melanocytes among bleached granules. The nuclei of melanocytes are almost imperceptible, consisting of a thin nuclear envelope and with almost no stainable chromatin (bar $=30 \mu \mathrm{m})$.

documented only sporadically, heterotopic melanocytes may also be found in ipsilateral optic nerve sheath, soft tissue of the orbit, and meningeal covering of the brain. Ectopic melanocytes in these locations can rarely give rise to melanoma. However, the increased density of melanocytes within the iris, ciliary body, and choroid places persons with oculodermal melanocytosis at considerably greater lifetime risk of uveal melanoma [6].

Of 33 patients with primary orbital melanoma reported in the literature up to 2002, 27 cases (82\%) arose tion reveals the wispy, shredded wheat appearance of the collagen in longitudinal section ( $\mathrm{bar}=80 \mu \mathrm{m})$. The right panel shows splayed and fragment collagen that makes up the fascicles. The degenerative changes impart weak eosin uptake (bar $=35 \mu \mathrm{m})$. in the context of either oculodermal melanocytosis or blue nevus type of malformation $[7,8]$. In our patient, the orbital melanoma was found adjacent to benign melanocytes, the presumed cells that spawned the malignancy.

To our knowledge, melanoma-associated spongiform scleropathy has not been reported in an eye without a contiguous choroidal or ciliary body melanoma. The characteristic degenerative changes in this case were present in the inner sclera adjacent to a diffuse choroidal and ciliary body nevus (typical uveal nevus component of oculodermal melanocytosis), a diagnosis confirmed by careful inspection of bleached sections.

In the most comprehensive paper on the subject of melanoma-associated spongiform scleropathy, the scleral disorder was defined by light microscopic criteria alone using hematoxylin-eosin stains [1]. Although that report documented increased total glycosaminoglycan within the affected sclera, this was not correlated with any histochemical stain. In another paper, nonspecific stains for glycosaminoglycan (periodic acid-Schiff reaction, alcian blue, and colloidal iron) were reported as diminished in areas of scleral degeneration [2]. Reduced staining intensity was attributed to dilution of ground substance in the affected areas, or as described by the investigators 'an accumulation of a material that has no specific staining characteristics with the particular stainings [sic] applied' [2]. This is consistent with our findings of variable to diminished staining with alcian blue and colloidal iron. To our knowledge, there has been no previously published illustration showing the staining properties of these stains in melanoma-associated sponiform scleropathy. 
The implications of the finding in this case are unknown, as the mechanism(s) and clinical significance of melanoma-associated spongiform scleropathy are partially understood [9]. There is evidence that the collagen changes in melanoma-associated spongiform scleropathy may predispose to scleral invasion and possibly contribute to a less favorable outcome [1-4]. However, in our case, the scleral changes did not arise next to a melanoma, but rather adjacent to a large, heavily pigmented nevus, which had been present from birth (or at least at a very young age). Melanoma-associated spongiform scleropathy is not associated with tumor pigmentation but is positively correlated with older patient age [1]. One could speculate that prolonged contact with the sclera, as in the case of a congenital melanocytic uveal nevus, may give rise to the spongiform changes seen in the sclera. If this is true, scleropathy should be anticipated in other eyes with melanosis oculi.

The majority of research on melanoma-associated spongiform scleropathy has come from a single institution, and the finding has rarely been reported by others $[1,2,9]$. The pre-enucleation ultrasound biomicroscopic features of melanoma-associated spongiform scleropathy have been reported once [10]. We are not aware of this type of spongiform scleral degeneration occurring in any other disorder than in choroidal and ciliary body melanoma. The histologic differential diagnosis includes fixation or cutting artifact that might give collagen a loose, fibrillary appearance. Other forms of scleral degeneration, such as focal scleral mucinosis and scleropachynsis, display increased staining with mucin stains rather than diminished staining as found in melanoma-associated spongiform scleropathy $[3,11,12]$. Given that this scleropathy is a histologic diagnosis and the clinical implications are unclear, it is possible that this type of degenerative collagen process has been underreported or overlooked in eyes harboring other disorders.

\section{Statement of Ethics}

The subject gave consent for use of material in this report.

\section{Disclosure Statement}

The authors have no conflicts of interest to disclose and concur on the contents for this submission.

\section{References}

1 Alyahya GA: Melanoma-associated spongiform scleropathy: characterization, biochemical and immunohistochemical studies. Acta Ophthalmol 2008;86:1-21.

2 Alyahya GA, Heegaard S, Prause JU: Characterization of melanoma associated with spongiform scleropathy. Acta Ophthalmol Scand 2002;80:322-326

3 Robert F: The sclera; in Heegaard S, Grosssniklaus HE (eds): Eye Pathology. Heidelberg, Spinger, 2015, chapt 4, pp 155-172.

4 Alyahya GA, Ribel-Madesen SM, Heegaard S, Prause JU, Trier K: Melanoma-associated spongiform scleropathy: biochemical changes and possible relation to tumour extension. Acta Ophthalmol Scand 2003;81:625-629.
5 Margo CE: Pigmented lesions of the eyelid; in Albert DM, Jakobiec FA (eds): Principle and Practice of Ophthalmology, ed 2. Philadelphia, Saunders, 2000, vol 4, chapt 257, pp 3430-3446.

6 Singh AD, De Potter P, Fijal BA, Shields CL, Shields JA, Elston RC: Lifetime prevalence of uveal melanoma in white patients with oculo(dermal) melanocytosis. Ophthalmology $1998 ; 105: 195-198$.

7 Tellada M, Specht CS, McLean IW, Grossniklaus HE, Zimmerman LE: Primary orbital melanoma. Ophthalmology 1996;103:929932.

8 Lee V, Sandy C, Rose GE, Moseley IM, Cree I, Hungerford JL: Primary orbital melanoma masquerading as vascular anomalies. Eye 2002;16:16-20.
9 Alyahya GA, Kolko M, Prause JU, et al: Matrix matalloproteinase-2 is expressed in melanoma-associated spongiform scleropathy. Invest Ophthalmol Vis Sci 2008;49:2806-2811.

10 Weisbrod D, Pavlin CJ, McGowan H, Yucel $\mathrm{YH}$ : Clinical detection of melanoma-associated spongiform scleropathy by ultrasound biomicroscopy and its correlation with pathological diagnosis. Arch Ophthalmol 2009;127: 1064-1066.

11 Margo CE: Focal scleral mucinosis. Arch Ophthalmol 1986;104:806-807.

12 Conn H, Green WR, de la Cruz ZC, Hillis A: Scleropachynsis maculopathy. Arch Ophthalmol 1982;100:793-799. 